\title{
Fabrication of porous ethyl cellulose microspheres based on the acetone-glycerin-water ternary system: Controlling porosity via the solvent-removal mode
}

\author{
Masahiro Murakami* , Akihiro Matsumoto, Chie Watanabe, Yu Kurumado, Masashi Takama \\ Laboratory of Pharmaceutics, Faculty of Pharmacy, Osaka Ohtani University, Osaka, Japan.
}

\begin{abstract}
Summary Porous ethyl cellulose (EC) microspheres were prepared from the acetone-glycerin-water ternary system using an oil/water $(\mathrm{O} / \mathrm{W})$-type emulsion solvent extraction method. The $\mathbf{O}$ / W type emulsion was prepared using acetone dissolved ethyl cellulose as an oil phase and aqueous glycerin as a water phase. The effects of the different solvent extraction modes on the porosity of the microspheres were investigated. The specific surface area of the porous EC microspheres was estimated by the gas adsorption method. When the solvent was extracted rapidly by mixing the emulsion with water instantaneously, porous EC microspheres with a maximum specific surface area of $40.7 \pm 2.1 \mathrm{~m}^{2} / \mathrm{g}$ were obtained. On the other hand, when water was added gradually to the emulsion, the specific surface area of the fabricated microspheres decreased rapidly with an increase in the infusion period, with the area being $\mathbf{2 5 - 4 5 \%}$ of the maximum value. The results of an analysis of the ternary phase diagram of the system suggested that the penetration of water and glycerin from the continuous phase to the dispersed phase before solidification affected the porosity of the fabricated EC microspheres.
\end{abstract}

Keywords: Porous microspheres, ethyl cellulose, acetone-glycerin-water ternary system, phase separation, solvent extraction

\section{Introduction}

Polymeric microspheres have been investigated widely for use as drug delivery systems. For instance, they can be used for the oral (1-5) and pulmonary delivery of drugs $(6)$ and as injectable carriers for long-term drug release (7). Among the various types of polymeric microspheres being studied, porous microspheres are attracting the greatest attention with respect to pulmonary drug delivery. Owing to their high porosity, porous microspheres exhibit aerodynamic diameters smaller than their geometric ones. The relationship between their aerodynamic and geometric diameters can be expressed as follows:

$$
\mathrm{D}_{\text {aero }}=\mathrm{D}_{\text {geo }}\left(\mathrm{P} / \mathrm{P}_{\text {ref }} \cdot \mathrm{R}\right)^{1 / 2}
$$

where $\mathrm{D}_{\text {aero }}$ is the aerodynamic diameter, $\mathrm{D}_{\text {geo }}$ is the

\footnotetext{
*Address correspondence to:

Dr. Masahiro Murakami, Osaka Ohtani University, 3-11-1

Nishikori-kita, Tondabayashi, Osaka 584-0854, Japan.

E-mail: murakam@osaka-ohtani.ac.jp
}

geometric diameter, $\mathrm{R}$ is the shape factor ( $\mathrm{R}$ is 1 for truly spherical particles), $\mathrm{P}$ is the particle density, and $\mathrm{P}_{\text {ref }}$ is the reference (water) density. The aerodynamic diameter of inhaled particles is one of the primary factors determining the distribution of the particles in the lung. Hence, microspheres with controlled porosity can be delivered readily to the target area in the lung, because the aerodynamic diameter of microspheres can be controlled by varying their porosity.

Researchers have proposed a number of methods for fabricating porous polymeric microspheres. Chai and coworkers have reviewed that most preparation methods are one of the following types: the solvent evaporation method, the polymerization method, the seedswelling method, the synthesis method, the sintering method, the phase-separation method, and the spraydrying method (8). Of these, the solvent evaporation method based on the use of multiple emulsions and, in particular, water-in-oil-in-water $(\mathrm{W} / \mathrm{O} / \mathrm{W})$ emulsions, is used commonly for fabricating porous microspheres. During the W/O/W-type emulsion solvent evaporation method, pores are formed when water migrates from the internal water phase to the external water phase of 
the emulsion. Porogens, which are pore-forming agents, are often added to the dispersed phase for controlling the porosity. Previous studied have used ammonium bicarbonate, sodium chloride, gelatin, paraffin, canola oil, and glycerol monooleate as porogens (9-11).

We had previously proposed a new method for fabricating polymeric microspheres without using halogenated solvents (12). The method was based on the phase separation of acetone and glycerin; the dispersed and continuous phases used were poly(lactide-coglycolide) in acetone and aqueous glycerin, respectively. We had demonstrated that the encapsulation efficiency and drug release profile of vitamin- $B_{12}$-loaded microspheres fabricated using this method were similar to those of microspheres produced by the conventional method using methylene chloride. We had also found that the water content of the continuous phase affected the formation of the microspheres. Although the biphasic area in the low-water-content section of the triangular diagram of the acetone-glycerin-water ternary system was small, we had found that microspheres formed in and near the biphasic area. In contrast, microspheres did not form in the high-water-content area (i.e., the monophasic area). However, even though we had demonstrated the phenomenon of emulsification in the ternary system, the process of removal of the solvents from the resultant emulsion was not fully examined.

During the solvent-extraction process, the migration of the porogens can directly lead to the formation of pores. Further, the water and glycerin in the continuous phase can probably act as porogens for porous ethyl cellulose (EC) microspheres, because these materials are poor solvents of EC. Therefore, the flux of water and glycerin between the dispersed and continuous phases probably plays a key role in determining the porosity of the fabricated EC microspheres. Based on this hypothesis, we propose that the porosity of EC microspheres fabricated using the acetone-glycerinwater ternary system can be controlled by varying the mode by which the emulsion and water flow into each other during the solvent-extraction process. Thus, in the present study, we investigated the effects of the solventextraction process on the formation of pores on the surfaces of the EC microspheres fabricated from the acetone-glycerin-water ternary system.

\section{Materials and Methods}

\subsection{Materials}

The EC sample (7 cps) used in this study was donated by Nisshin kasei Co., Ltd (Osaka, Japan). All the other chemicals used were of reagent grade.

\subsection{Fabrication of EC microspheres}

i) Fabrication without using halogenated solvents The method for fabricating EC microspheres without using halogenated solvents is illustrated in Figure 1. First EC $(2.0 \mathrm{~g})$ was dissolved in acetone $(16 \mathrm{~g})$ to obtain the dispersed phase. The dispersed phase was emulsified in a $5 \%$ aqueous solution of polyvinyl alcohol (PVA, Kuraray Co., Ltd., Osaka, Japan ) (7.5 g)-glycerin (17.5 g) by mixing using a propeller mixer (HEIDON Threeone moter BL1200, Shinto Scientific Co., Ltd., Tokyo, Japan) for $1 \mathrm{~min}$ at $300 \mathrm{rpm}$ at room temperature. The resultant emulsion was mixed with $500 \mathrm{~mL}$ of water in two modes: (a) the resultant emulsion was mixed in 500 $\mathrm{mL}$ of water instantaneously (mode 1 in Figure 1) or (b) $500 \mathrm{~mL}$ of water was added slowly to the resultant emulsion and mixed at $400 \mathrm{rpm}$ for a certain period (mode 2). The resulting EC microspheres, which were hard, were washed with deionized water. Next, the aggregated EC microspheres were removed by filtration using $75-\mu \mathrm{m}$ and $53-\mu \mathrm{m}$ sieves, and the nonaggregated ones were collected by filtration with a $20-\mu \mathrm{m}$ sieve. The EC microspheres were the redispersed in deionized water and lyophilized.

ii) Fabrication by the conventional $\mathrm{O} / \mathrm{W}$-type emulsion solvent evaporation method using a halogenated solvent First, EC (1.0 g) was dissolved in dichloromethane (14 $\mathrm{mL})$ to obtain an oil phase. The oil phase was emulsified in a $0.5 \%$ PVA solution $(20 \mathrm{~mL})$ using a homogenizer (Polytron ${ }^{\circledR}$ PT3100, Kinematica Ag, Luzern, Switzerland) for $5 \mathrm{~min}$ at 4,000 rpm at room temperature. The resultant emulsion was added to $400 \mathrm{~mL}$ of water all at once and stirred at $400 \mathrm{rpm}$ at room temperature for $3 \mathrm{~h}$, in order to remove the dichloromethane. The obtained hardened EC microspheres were washed with deionized water. Finally, the aggregated EC microspheres were removed by filtration using $75-\mu \mathrm{m}$ and $53-\mu \mathrm{m}$ sieves, and the nonaggregated ones were collected by filtration with a 20 $\mu \mathrm{m}$ sieve. The EC microspheres were then redispersed in

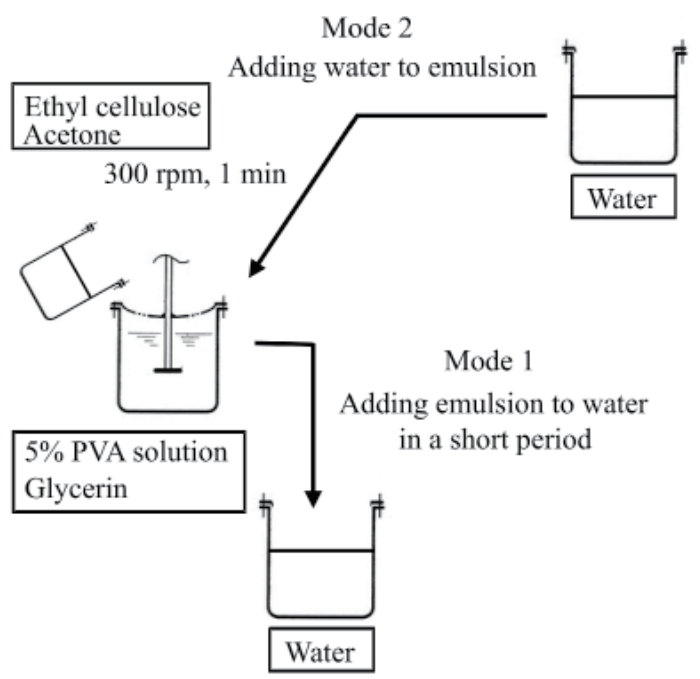

Figure 1. Illustration of the proposed method for preparing EC microspheres. In mode 1 , the emulsion is added to water instantaneously. In mode 2, water is added to the emulsion for $1 \mathrm{~min}, 3 \mathrm{~min}, 5 \mathrm{~min}, 10 \mathrm{~min}$, or $20 \mathrm{~min}$. 
deionized water and lyophilized.

iii) Fabrication by the conventional W/O/W-type emulsion solvent evaporation method using a halogenated solvent First, EC $(1.0 \mathrm{~g})$ was dissolved in dichloromethane $(14 \mathrm{~mL})$ to obtain an oil phase. Then, a $0.5 \%$ PVA solution $(1 \mathrm{~mL})$ was emulsified into the oil phase using a homogenizer (T18 Digital ULTRA-TURRAX ${ }^{\mathbb{B}}$, IKA Works GmbH \& Co. KG, Staufen im Breisgau, Germany) for $30 \mathrm{~s}$ at $25,000 \mathrm{rpm}$ at room temperature. The obtained $\mathrm{W} / \mathrm{O}$ emulsion was mixed in a $0.5 \%$ PVA solution $(20 \mathrm{~mL})$ using the homogenizer for 5 $\min$ at $4,000 \mathrm{rpm}$ at room temperature. The resultant emulsion was added to $400 \mathrm{~mL}$ of water all at once and stirred at $400 \mathrm{rpm}$ at room temperature for $3 \mathrm{~h}$, in order to remove the dichloromethane. The hardened EC microspheres were washed. Next, the aggregated EC microspheres were removed by filtration using 75$\mu \mathrm{m}$ and $53-\mu \mathrm{m}$ sieves and the nonaggregated ones were collected by filtration with a $20-\mu \mathrm{m}$ sieve. Finally, the EC microspheres were redispersed in deionized water and lyophilized.

\subsection{Microscopy observations}

The fabricated EC microspheres were observed using a scanning electron microscopy (SEM) system (JSM5500 LV, JOEL Ltd., Tokyo, Japan). For the SEM observations, samples of the EC microspheres were prepared by depositing gold-palladium at $15 \mathrm{~mA}$ for 3 min (Quick Auto Coater JFC-1500, JOEL Ltd.).

\subsection{Determination of sizes of EC microspheres}

The sizes of the fabricated EC microspheres were determined using a laser diffraction particle size analyzer (SALD-2200, Shimadzu Co. Ltd., Kyoto, Japan).

\subsection{Determination of specific surface areas of EC microspheres}

The specific surface areas of the fabricated EC microspheres were determined by the gas adsorption method (FlowSorb 2310, Shimadzu Co., Ltd.).

\subsection{Analysis of triangular diagram}

The triangular diagram for the acetone-glycerinwater ternary system was taken from the literature. We determined the ratio of the upper phase (i.e., the acetone-rich phase) to the lower phase (i.e., the glycerin-rich phase) (w/w) and the composition of the upper phase in the phase-separated solution of the acetone-glycerin-water ternary system. The glycerin content was considered to be the residual constant weight after drying in vacuum. The water content was determined by the Karl Fisher method; a volume titrator a)

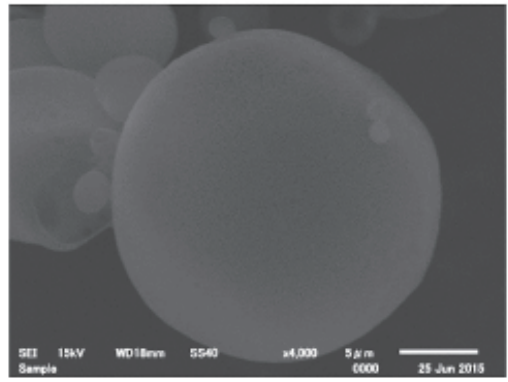

b)

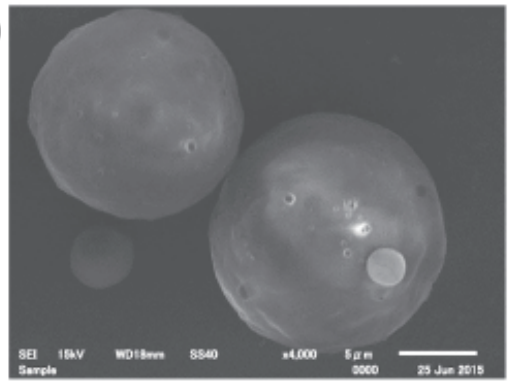

c)

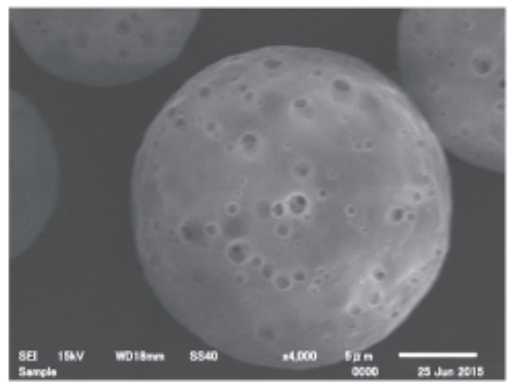

Figure 2. Scanning electron micrographs of the fabricated EC microspheres. The EC microspheres shown were prepared by (a) the proposed method (mode 1), (b) the conventional $\mathrm{O} / \mathrm{W}$-type emulsion solvent evaporation method, and (c) the conventional W/O/W-type emulsion solvent evaporation method. The bars represent $5 \mu \mathrm{m}$.

(AQV-300, Hiranuma Sangyo, Co., Ltd., Ibaragi, Japan) was employed for the purpose.

\section{Results}

\subsection{Morphological properties and particle sizes of fabricated EC microspheres}

Micrographs of the fabricated EC microspheres are shown in Figure 2. No micron-sized pores were observed on the surfaces of the EC microspheres fabricated using the new method. On the other hand, a large number of micron- and submicron-sized pores were observed on the surfaces of the EC microspheres fabricated by the conventional W/O/W-type emulsion solvent evaporation method. It should be noted that the EC microspheres fabricated using the conventional $\mathrm{O} /$ W-type emulsion solvent evaporation method also had micron- and submicron-sized pores on their surfaces; however, these were fewer in number.

The size distributions of the EC microspheres obtained using the three different methods are shown in Figure 3. The EC microspheres fabricated by the new 


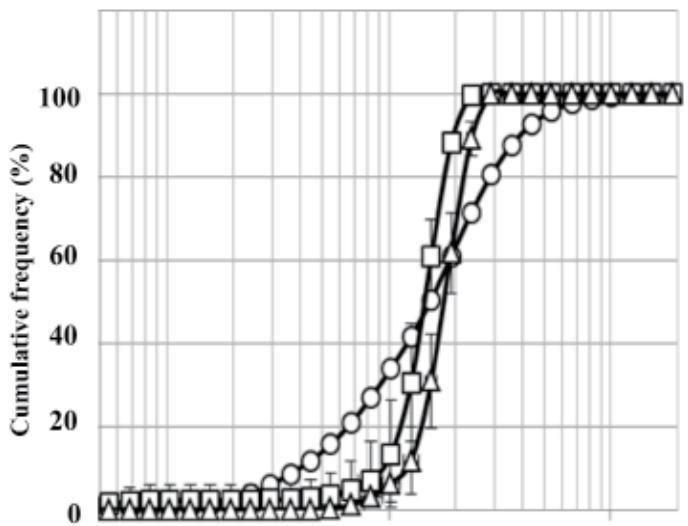

Figure 3. Weight and size distributions of the fabricated EC microspheres. $\circ$ : proposed method (mode 1), $\square$ : conventional $\mathrm{O} / \mathrm{W}$-type emulsion solvent evaporation method, $\Delta$ : conventional $\mathrm{W} / \mathrm{O} / \mathrm{W}$-type emulsion solvent evaporation method. The data shown represent the mean \pm standard deviation (SD) ( $n=3$ batches).

Table 1. The specific surface area of microspheres prepared by the new and conventional methods

\begin{tabular}{lc}
\hline Method & Specific surface area $\left(\mathrm{m}^{2} / \mathrm{g}\right)$ \\
\hline $\begin{array}{l}\text { New method } \\
\quad \text { mode 1 }\end{array}$ & $40.7 \pm 2.10$ \\
$\begin{array}{l}\text { Conventional method } \\
\text { O/W-type solvent evaporation }\end{array}$ & $3.12 \pm 0.19$ \\
$\quad$ W/O/W-type solvent evaporation & $5.73 \pm 0.63$ \\
\hline
\end{tabular}

The data represent the means $\pm \mathrm{SD}(\mathrm{n}=3$ batches $)$.

method had a broader particle-size distribution than did those produced using the two conventional method. The mean particle sizes of the EC microspheres fabricated by the new method, the conventional $\mathrm{O} /$ W-type emulsion solvent evaporation method, and the conventional W/O/W-type emulsion solvent evaporation method were $13.7 \pm 0.5,13.3 \pm 2.6$, and $17.2 \pm 1.5 \mu \mathrm{m}$, respectively (mean \pm standard deviation for 3 batches). In the case of the EC microspheres fabricated by the new method, there were no significant changes in the mean particle size or the size distribution with changes in the solvent-infusion mode and the duration of infusion during the solvent-extraction process.

\subsection{Specific surface areas of fabricated EC microspheres}

As shown in Table 1, the specific surface areas of the EC microspheres fabricated using the three methods could be arranged in the following order: the new method (mode 1$)>$ the conventional $\mathrm{W} / \mathrm{O} /$ W-type emulsion solvent evaporation method $>$ the conventional $\mathrm{O} / \mathrm{W}$-type emulsion solvent evaporation method. In the case of the new method, the mode of solvent extraction had an effect on the specific surface area of the obtained EC microspheres (Figure 4.). Mode 1 resulted in the highest specific surface area, which was $40.7 \mathrm{~m}^{2} / \mathrm{g}$. In contrast, mode 2 resulted in specific

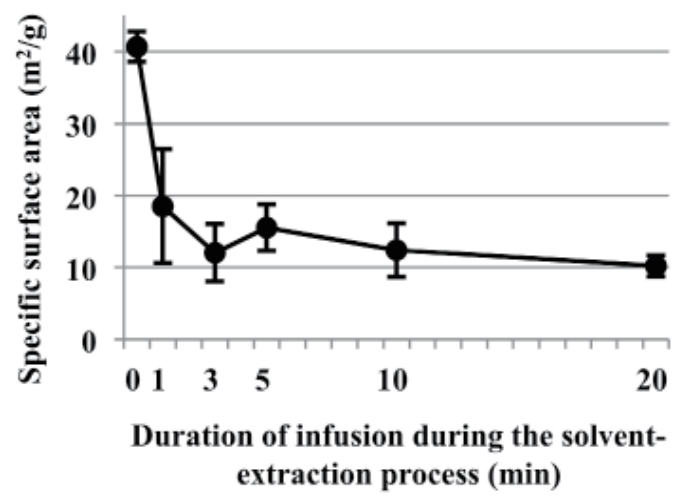

Figure 4. Effects of the infusion period during the solventextraction process on the specific surface area of the microspheres. The point at time 0 indicates the specific surface area of the EC microspheres prepared in mode 1. The points at other times indicate the specific surface areas of the EC microspheres prepared in mode 2 . The data shown represent the mean $\pm \mathrm{SD}(n=3$ batches $)$.

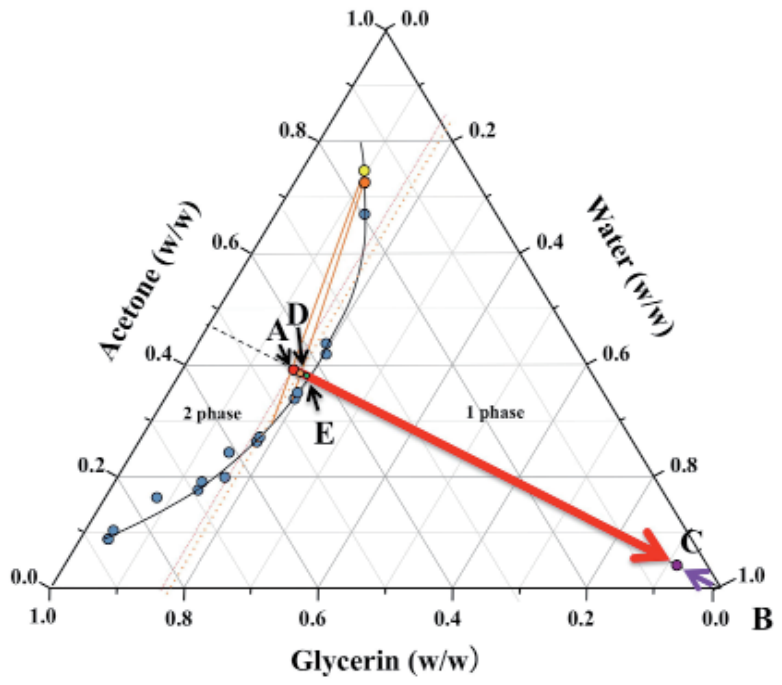

Figure 5. Triangular diagram of the acetone-glycerinwater ternary system. The data corresponding to the border line between phases 1 and 2 were taken from the literature (12). The concentrations of acetone, glycerin, and water for the various points were as follows: point A (acetone/glycerin/ water $=0.399: 0.424: 0.178)$, point $\mathrm{D}$ (acetone/glycerin/ water $=0.388: 0.424: 0.188$ ), and point $\mathrm{E}$ (acetone/glycerin/water $=$ 0.386:0.422:0.193).

surface areas of $10.2-18.5 \mathrm{~m}^{2} / \mathrm{g}$; further, in this case, the specific surface area decreased with an increase in the infusion period.

\subsection{Analysis of triangular diagram}

The triangular diagram of the acetone-glycerin-water ternary system is shown in Figure 5. Point A represents the status of the emulsion formed after the completion of the emulsification stage in the new method. Point $\mathrm{C}$ represents the status of emulsion at the end of the solvent-extraction process. The purple and red arrows represent the transitions during the solvent- 
Table 2. Phase ratio and composition of the acetone-rich phase at each point in Figure 5

\begin{tabular}{|c|c|c|c|c|}
\hline \multirow{2}{*}{ Items } & \multirow{2}{*}{ Ratio of acetone-rich phase } & \multicolumn{3}{|c|}{ Composition of acetone-rich phase (w/w \%) } \\
\hline & & Water content & Glycerin content & Acetone content \\
\hline Point A & 0.110 & 10.5 & 14.8 & 74.7 \\
\hline Point D & 0.033 & 11.5 & 15.7 & 72.8 \\
\hline Point E & & & miscible & \\
\hline
\end{tabular}

Composition: point A (acetone/glycerin/water = 0.399:0.424:0.178), point D (acetone/glycerin/water $=0.388: 0.424: 0.188$ ), point E (acetone/ glycerin/water $=0.386: 0.422: 0.193)$.

extraction process. For the case where the solvent was extracted rapidly by mixing the emulsion with water instantaneously, the status of emulsion is represented as the jump from point $\mathrm{A}$ to $\mathrm{B}$ and the transition from point $\mathrm{B}$ to $\mathrm{C}$ on the purple arrow (mode 1 ). The purple arrow is in the monophasic area. For the case where water was added gradually to the emulsion, the status is represented as the transition from point $\mathrm{A}$ to $\mathrm{C}$ through points $\mathrm{D}$ and $\mathrm{E}$ on the red arrow (mode 2). The amount ratios $(\mathrm{w} / \mathrm{w})$ and compositions of the acetone-rich phase for points $\mathrm{A}, \mathrm{D}$, and $\mathrm{E}$ of the triangular diagram are listed in Table 2. The amount ratio of the acetone-rich phase at point $\mathrm{D}$ in the ternary system was $30 \%$ of that at point $\mathrm{A}$, even though only a small amount of water was added. On the other hand, the water and glycerin contents of the acetone-rich phase for the two points were similar. At point E, the ternary system transitioned into the monophasic area.

\section{Discussion}

In a previous report, we had introduced a new method for fabricating sustained-release microspheres using nonhalogenated solvents. This method was based on the phase separation of acetone and aqueous glycerin (12). In this study, we evaluated the feasibility of using this method for fabricating porous EC microspheres. For the purposes of evaluation, we selected the morphological properties and the specific surface area of the fabricated EC microspheres, which is an indicator of their porosity, as the evaluation parameters. Elucidating the morphological properties of porous microspheres is essential for determining their applicability in various fields. Similarly, being able to control the porosity of the microspheres is crucial for adjusting their target profile, that is, for adjusting their release profile with respect to oral and pulmonary drug delivery.

First, we determined the morphological properties and specific surface areas of the fabricated EC microspheres and compared them with those of the microspheres fabricated by the two conventional methods. SEM observations revealed that the surface morphologies of the EC microspheres fabricated by the new method and those of the microspheres produced by the conventional $\mathrm{O} / \mathrm{W}$-type emulsion solvent evaporation method were similar. The fabricated EC microspheres had smooth surfaces; this was in keeping with previously obtained results (12). On the other hand, the specific surface areas of the EC microspheres prepared by the new and conventional methods were different. The EC microspheres fabricated by the new method (mode 1) had a specific surface area that was approximately 13.0 times than of the microspheres produced by the conventional $\mathrm{O} / \mathrm{W}$-type emulsion solvent evaporation method. Further, the specific surface area of the microspheres produced by the new method was 7.1 times than of the microspheres produced by the conventional $\mathrm{W} / \mathrm{O} /$ W-type emulsion solvent evaporation method; in the latter case, the microspheres had pores on their surfaces. These differences cannot be explained merely by the differences in the particle-size distribution of the EC microspheres, because the microspheres prepared by the new and conventional methods had similar mean sizes.

The formation of pores on the EC microspheres prepared by the conventional methods can be explained as follows. It is known that water exhibits low solubility in dichloromethane, which is used commonly as a solvent for the oil phase in the conventional preparation methods. This means that water cannot penetrate into the oil phase from the external water phase in the case of the conventional methods. Therefore, pore formation is suppressed in the case of the conventional O/W-type emulsion solvent evaporation method. Moreover, in the case of the conventional $\mathrm{O} / \mathrm{W}$-type emulsion solvent evaporation method, the amount of porogens in the oil phase was low. Hence, the resulting microspheres had a lower specific surface area. On the other hand, in the case of the conventional $\mathrm{W} / \mathrm{O} / \mathrm{W}$-type emulsion solvent evaporation method, the inner water phase played the role of a porogen, resulting in the fabrication of porous EC microspheres. Hence, the conventional W/O/ W-type emulsion solvent evaporation method resulted in microspheres with a specific surface area twice that of the microspheres produced using the conventional $\mathrm{O} /$ W-type emulsion solvent evaporation method.

In the case of the new method, the solvents for the dispersed and external phases were acetone and aqueous glycerin, respectively. The mutual solubility of acetone and aqueous glycerin depends on the concentrations in which these three components, namely, acetone, glycerin, and water, are present in the system. Because aqueous glycerin is a poor solvent of 
ethyl cellulose, it can act as a porogen for the synthesis of porous EC microspheres. In the condition of high mutual solubility of acetone and aqueous glycerin, such solvents penetrated in the dispersed phase from the continuous phase result in the formation of pores in EC microspheres when removed during the solventextraction process. Hence, the resultant microspheres had a high specific surface area.

Next, we investigated whether it was possible to control the specific surface area on the basis of the flux of glycerin and water into the dispersed phase. It was found that the mode of infusion during the solvent-extraction process determined the specific surface area of the fabricated porous EC microspheres. This phenomenon can be explained on the basis of the triangular diagram of the acetone-glycerin-water ternary system (Figure 5). In the emulsification stage, the ternary system is at point $\mathrm{A}$. When the solvent is extracted rapidly by adding the emulsion to water instantaneously (mode 1), the ternary system jumps from point $A$ to point $B$ and then from point $B$ to point $C$. From point $B$ to point $C$, the ternary system is in the monophasic area. Thus, acetone, water, and glycerin are assumed to dissolve mutually in the dispersed phase, before the solidification of the EC microspheres. Therefore, EC microspheres with a high specific surface area could be formed using the new method.

On the other hand, the specific surface area of the obtained EC microspheres decreased when water was added gradually to the emulsion (mode 2). This phenomenon can be explained on the basis of the change in the amount of porogens in the dispersed phase as follows. During the emulsification process, the concentrations of water and glycerin in the dispersed phase reached $10.5 \%$ and $14.8 \%$ (point A), respectively. Further, the concentrations of water and glycerin in the acetone-rich phase remained almost constant as water was added ( $\mathrm{A} \rightarrow \mathrm{D}$ transition). The same phenomenon probably also occurred during the $\mathrm{D} \rightarrow \mathrm{E}$ transition. However, the volume of the acetonerich phase decreased markedly ( $\mathrm{A} \rightarrow \mathrm{D} \rightarrow \mathrm{E}$ transition). This indicated that the solvents in the dispersed phase were extracted into the continuous phase without there being an increase in the amounts of the porogens (water and glycerin) before the solidification of the EC microspheres. Thereafter, the porogens were unable to penetrate into the $\mathrm{EC}$ microspheres even when more water was added in the monophasic region $(\mathrm{E} \rightarrow \mathrm{C})$ because the $\mathrm{EC}$ microspheres had already solidified. Hence, the formation of additional pores was suppressed.

The above-mentioned results confirmed that the proposed method based on the acetone-glycerin-water ternary system was suitable for fabricating porous EC microspheres. Next, we discuss the potential uses of the porous EC microspheres prepared using the new method. Porous materials can be classified on the basis of their pore size, and the applicability of porous microspheres depends on their pore size. Microspheres with micrometer-sized pores are being explored for use in tissue engineering, because such microspheres can be used as scaffolds for cell growth $(10,13)$. On the other hand, mesoporous $(2-50 \mathrm{~nm})$ microspheres are being investigated for use for controlled drug release, owing to their larger specific surface area $(1-3,5)$. Since we did not measure the sizes of the pores of the fabricated EC microspheres in this study, it is not possible to comment on their use. However, it is likely that the microspheres fabricated using the new method were mesoporous, judging from their electron micrographs and their specific surface area, which was measured using the gas adsorption method. Microspheres with large specific surface areas, such as the ones fabricated using the new method, can be used for the controlled release of drugs. Further, such microspheres can be used as carriers for pulmonary drug delivery, owing to their large specific surface area. Various uses for the microspheres fabricated using the new method are being explored in our laboratory.

\section{Acknowledgements}

The authors thank Ms. Kayoko Murakami and Mr. Kohsuke Horita for their technical assistance. We would like to thank Editage (www.editage.jp) for English language editing.

\section{References}

1. Vaghani S, Vasan ti S, Chaturvedi K, Satish CS, Jivani NP. Stomach-specific drug delivery of 5-Fluorouracil using ethyl cellulose floating microspheres. Pharm Devel Tech. 2010; 15:154-161.

2. Tozuka Y, Sugiyama E, Takeuchi H. Release profile of insulin entrapped on mesoporous materials by freezethaw method. Int J Pharm. 2010; 386:172-177.

3. Zhang Y, Zhi Z, Jiang T, Zhang J, Wang Z, Wang S. Spherical mesoporous silica nanoparticles for loading and release of the poorly water-soluble drug telmisartan. J Cont Rel. 2010; 145:257-263.

4. Bhise SB, More AB, Malayandi R. Formulation and in vitro evaluation of rifampicin loaded porous microspheres. Sci Pharm. 2010; 78:291-302.

5. Kapoor S, Hegde R, Bhattacharyya AJ. Influence of surface chemistry of mesoporous alumina with wide pore distribution on controlled drug release. J Cont Rel. 2009; 140:34-39.

6. Arnold MA, Gorman EM, Schieber LJ, Munson EJ, Berland C. NanoCipro encapsulation in monodisperse large porous PLGA microparticles. J Cont Rel. 2007; 121:100-109.

7. Ogawa Y, Yamoto M, Takada S, Okada H, Shimamoto T. Controlled-release of leuprolide acetate from polylactic acid or copoly(lactic/glycolic) acid microcapsules: Influence of molecular weight and copolymer ratio of polymer. Chem Pharm Bull. 1988; 36:1502-1507. 
8. Cai Y, Chen Y, Hong X, Liu Z, Yuan W. Porous microsphere and its applications. Int J Nanomed. 2013; 8:1111-1120.

9. Kim TK, Yoon JJ, Lee DS, Park TG. Gas foamed open porous biodegradable polymeric microspheres. Biomaterials. 2006; 27:152-159.

10. Draghi L, Resta S, Pirozzolo MG, Tanzi MC. Microspheres leaching for scaffold porosity control. J Mater Sci Mater Med. 2005; 16:1093-1097.

11. Ahmed AR, Bodmeier R. Preparation of preformed porous PLGA microparticles and antisense oligonucleotides loading. Eur J Pharm Biopharm. 2009;
71:264-270.

12. Matsumoto A, Kitazawa T, Murata J, Horikiri Y, Yamahara H. A novel preparation method for PLGA microspheres using non-halogenated solvents. J Cont Rel. 2008; 129:223-227.

13. Keskar V, Marion NW, Mao JJ, Gemeinhart RA. In vitro evaluation of microporous hydrogels to facilitate stem cell infiltration, growth and mineralization. Tissue Eng. 2009; 15:1695-1707.

(Received August 10, 2015; Revised August 25, 2015; Accepted August 26, 2015) 